\title{
Virus-induced gene silencing in the perennial woody Paeonia ostii
}

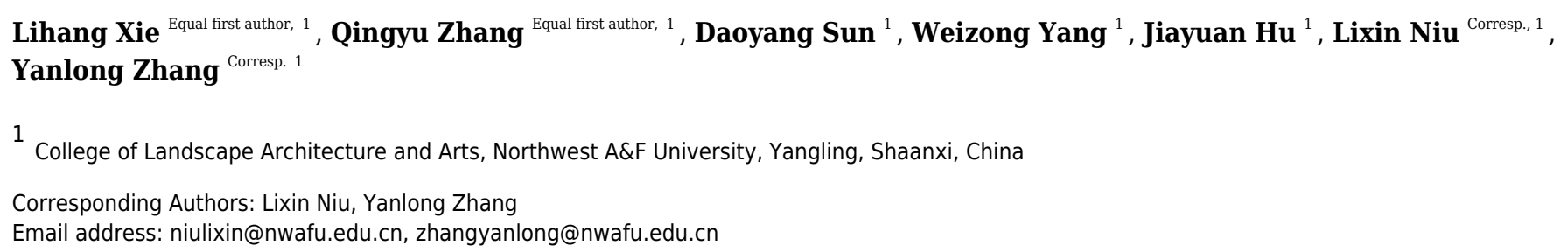

Tree peony is a perennial deciduous shrub with great ornamental and medicinal values. A limitation for its current functional genomic research is the lack of effective molecular genetic tools. Here, the first application of a Tobacco rattle virus (TRV)-based virus-induced gene silencing (VIGS) in the tree peony species Paeonia ostii is presented. Two different approaches, leaf syringe-infiltration and seedling vacuum-infiltration, were utilized for Agrobacterium-mediated inoculation. The vacuum-infiltration was shown to result in a more complete Agrobacterium penetration than syringe-infiltration, and thereby determined as an appropriate inoculation method. The silencing of reporter gene PoPDS encoding phytoene desaturase was achieved in TRV-PoPDS-infected triennial tree peony plantlets, with a typical photobleaching phenotype shown in uppermost newly-sprouted leaves. The endogenous POPDS transcripts were remarkably down-regulated in VIGS photobleached leaves. Moreover, the green fluorescent protein (GFP) fluorescence was detected in leaves and roots of plants inoculated with TRV-GFP, suggesting the capability of TRV to silence genes in various tissues. Taken together, the data demonstrated that the TRV-based VIGS technique could be adapted for high-throughput functional characterization of genes in tree peony. 
3 Lihang Xie† - Qingyu Zhang† - Daoyang Sun† $\cdot$ Weizong Yang $\cdot$ Jiayuan Hu $\cdot$ Lixin Niu* .

6 College of Landscape Architecture and Arts, Northwest A\&F University, Yangling, Shaanxi

7 712100, People's Republic of China; Oil Peony Engineering Technology Research Center of

8 National Forestry Administration, Yangling, Shaanxi, People’s Republic of China

$10 *$ To whom correspondence should be addressed:

11 Lixin Niu

12 College of Landscape Architecture and Arts, Northwest A\&F University, Yangling, Shaanxi,

13 People's Republic of China

14 Tel: +86-13572582039; Fax: +86-029-87082878; E-mail: niulixin@nwsuaf.edu.cn

15 Yanlong Zhang

16 College of Landscape Architecture and Arts, Northwest A\&F University, Yangling, Shaanxi,

17 People's Republic of China

18 Tel: +86-029-87082878; Fax: +86-029-87082878; E-mail: zhangyanlong@nwsuaf.edu.cn

† These authors contributed equally to this work. 


\section{Abstract}

25 Tree peony is a perennial deciduous shrub with great ornamental and medicinal values. A

26 limitation for its current functional genomic research is the lack of effective molecular genetic

27 tools. Here, the first application of a Tobacco rattle virus (TRV)-based virus-induced gene

28 silencing (VIGS) in the tree peony species Paeonia ostii is presented. Two different approaches,

29 leaf syringe-infiltration and seedling vacuum-infiltration, were utilized for Agrobacterium-

30 mediated inoculation. The vacuum-infiltration was shown to result in a more complete

31 Agrobacterium penetration than syringe-infiltration, and thereby determined as an appropriate

32 inoculation method. The silencing of reporter gene PoPDS encoding phytoene desaturase was

33 achieved in TRV-PoPDS-infected triennial tree peony plantlets, with a typical photobleaching

34 phenotype shown in uppermost newly-sprouted leaves. The endogenous PoPDS transcripts were remarkably down-regulated in VIGS photobleached leaves. Moreover, the green fluorescent protein (GFP) fluorescence was detected in leaves and roots of plants inoculated with TRV-GFP, suggesting the capability of TRV to silence genes in various tissues. Taken together, the data demonstrated that the TRV-based VIGS technique could be adapted for high-throughput functional characterization of genes in tree peony. 
45 Abbreviations

46 TRV Tobacco rattle virus

$47 \quad$ VIGS Virus-induced gene silencing

48 PTGS Post-transcriptional gene silencing

49 PDS Phytoene desaturase

50 EGFP Enhanced green fluorescent protein

51 qRT-PCR Quantitative real-time PCR

\section{Introduction}

54 Tree peony is a perennial woody plant belonging to sect. Moutan DC. of the genus Paeonia L.

55 (Paeoniaceae) (Li et al., 2009). It is indigenous to China and the cultivation history can be traced

56 back to 2000 years ago (Chen \& Li, 1998). As China's unofficial national flower, tree peony has

57 been introduced to Japan, America, Australia, and Europe, with a rise in worldwide popularity. It

58 is commonly known as an ornamental and medicinal crop due to large showy flowers and

59 abundant bioactive substances in roots. Recent reports suggest that the tree peony seed has high

60 yield of oil which contains over $90 \%$ unsaturated fatty acids required by human, revealing a

61 tremendous potential of tree peony in future edible oil production (Wu et al., 2014). The variety

62 Paeonia ostii 'Feng Dan' (P. ostii 'Feng Dan') is a new oil crop widely planted in north China, 
63

64

65

66

67

with its total cultivated area exceeding 16,200 hectares.

For now, a quantity of studies have been carried out on the cloning and function analysis of genes, associated with flower development (Li et al., 2016), bud dormancy (Zhang et al., 2015b), anthocyanin accumulation (Zhang et al., 2015a), and fatty acid biosynthesis (Yin et al., 2018), in tree peony. However, the conclusive studies on the function of genes in tree peony are tough because the lack of efficient genetic transformation system. Besides, the transgenic technology is time-consuming and laborious for the generation of homozygous lines, especially for plants with long life cycle like tree peony.

Virus-induced gene silencing (VIGS) is an attractively quick strategy for reverse genetic manipulation of non-model plants bypassing the stable transformation process (Ruiz et al., 1998; Burch-Smith et al. 2004). The VIGS experiment relies on the recombinant virus vector carrying an inserted partial sequence of a target plant gene to initiate RNA-mediated post-transcriptional gene silencing (PTGS), leading to transcript suppression of corresponding homologous gene (Baulcombe, 1999; Burch-Smith et al., 2004; Dinesh-Kumar et al., 2011). In this mechanism, double-stranded chimeric intermediates are first formed during viral replication in plant. Theses foreign intermediate are recognized and cleaved into 21-23 nucleotides of short interfering RNAs (siRNAs) by the enzyme DICER. Next, siRNAs are incorporated into the RNA-induced silencing complex (RISC) and target the complementary transcripts for cleavage, thus resulting in a specific degradation of host mRNA (Bartel, 2004; Senthil-Kumar \& Mysore, 2011). In contrast to gene silencing methods with inverted repeat sequences, VIGS has several advantages as simple plasmid assembly, short implementation cycle, and available identification of embryo- 
84

85

86

87

88

89

90

91

92

93

94

95

96

97

lethal genes (Reid et al., 2009).

Many viral vectors have been developed for VIGS assay, including Apple latent spherical virus (ALSV), Barely stripe mosaic virus (BSMV) (Holzberg et al., 2002), Cucumber mosaic virus (CMV) (Tasaki et al., 2016), Potato virus X (PVX) (Faivrerampant et al., 2004), Tobacco mosaic virus (TMV) (Kumagai et al., 1995), and Tobacco rattle virus (TRV) (Ratcliff et al., 2001). Compared to other viruses, TRV is capable of reaching apical meristem, inducing mild symptoms, and infecting wide range of plant species. Consequently, TRV vector has been widely used for silencing genes in a number of eudicots and monocots (Purkayastha \& Dasgupta, 2009), such as Arabidopsis (Burchsmith et al., 2006), tobacco (Liu et al., 2002), tomato (Quadrana et al., 2011), petunia (Sun et al., 2017), strawberry (Jia et al., 2011), rose (Wu et al., 2016), gladiolus (Singh et al., 2013), wheat, and maize (Zhang et al., 2017). At present, the VIGS technique is mostly applied to small herbaceous plants, and only a minority of woody plants achieves the set-up of VIGS system, like physic nut (Ye et al., 2009), grape (Kurth et al., 2012), and apple (Yamagishi \& Yoshikawa, 2013). The previous evidences indicate that tobacco rattle virus has been found in peony (Paeonia lactiflora 'Sarah Bernhardt') (Robertson et al. 2009). However, whether TRV-based VIGS can be applied to tree peony remains largely unknown.

Reporter gene is an essential component for indicating sites of silencing in VIGS system. PHYTOENE DESATURASE (PDS) is a key enzyme in the biosynthesis of protective carotene (Cunningham \& Gantt, 1998). Silencing of PDS results in characteristic photobleaching symptoms in infected plants (Stilio et al., 2010), and therefore it usually serves as a clear reporter. A modified TRV-GFP vector, bearing the coding region of enhanced green fluorescence protein 
105 (EGFP), also provides a visual tool for monitoring virus spread and silencing efficiency. This

106 vector has been successfully tested in several plants, including Arabidopsis, tobacco, rose,

107 strawberry, and chrysanthemum (Tian et al., 2014). In this study, we established an effective

108 VIGS system in P. ostii triennial seedlings by vacuum infiltration of TRV-PoPDS and TRV-GFP.

109 The upper systemically-infected leaves with TRV-PoPDS displayed a prominent photobleaching

110 phenotype and decreased PoPDS transcripts. GFP fluorescence was observed in TRV-GFP-

111 infiltrated leaves and roots under UV light irradiation. The data we have obtained demonstrated

112 the value of TRV-based VIGS for unraveling the functional significance of genes in tree peony.

114 Materials and Methods

115 Plant materials and growth conditions

116 Three-year-old seedlings of tree peony ( $P$. ostii 'Feng Dan') at four weeks post germination were

117 used for VIGS assay (Fig. 1a). The whole plant and leaves were agro-infiltrated with disposable

118 syringe and vacuum pressure for infection of TRV constructs, respectively. After inoculation, the

119 tree peony seedlings were rinsed with distilled water once and planted into plastic pots

120 containing a mixture of peat moss and vermiculite in a 3:1 volume ratio. Those plants were first

121 kept in the dark room at $15^{\circ} \mathrm{C}$ for one week, and then transferred into a growth chamber with a

$12216 \mathrm{~h} \mathrm{light/} 8 \mathrm{~h}$ dark photoperiod, and a day/night temperature range of $20 / 18{ }^{\circ} \mathrm{C}$. The inoculated

123 and uppermost systemically-infected leaves were used for phenotype observation, expression

124 profile analysis, and GFP fluorescence detection. 


\section{Isolation and sequence analysis of PoPDS}

127 Total RNA was extracted from the $P$. ostii 'Feng Dan' leaves with the TIANGEN RNA Prep

128 Pure Plat kit according to the manufacturer's recommendations (Tiangen, China). The first strand 129 of cDNA was synthesized using PrimeScript ${ }^{\circledR}$ RT reagent Kit with gDNA Eraser (Takara, Japan).

130 Primers were designed to amplify the PoPDS coding sequence based on transcriptome data during leaf development of P. suffruticosa Andrews (Luo et al., 2017). PCR was conducted using Taq DNA polymerase (Invitrogen, USA). The PCR reaction procedure was as follows: a cycle of $94{ }^{\circ} \mathrm{C}$ for $5 \mathrm{~min} ; 35$ cycles of $94{ }^{\circ} \mathrm{C}, 30 \mathrm{~s}, 54{ }^{\circ} \mathrm{C}, 30 \mathrm{~s}, 72{ }^{\circ} \mathrm{C}, 30 \mathrm{~s}$; a final cycle of $72{ }^{\circ} \mathrm{C}$ for 10 min. Next, the PCR products were cloned into the pUCm-T vector (TaKaRa, Japan). Positive clones were confirmed by DNA sequencing. Corresponding amino acids were deduced through the ExPASy translate tool (http://web.expasy.org/translate/). Multiple sequence alignment of

PoPDS with other similar proteins was performed by CLUSTALW (http://www.genome.jp/tools/clustalw/).

\section{Plasmid construction}

The TRV1, TRV2, and TRV2-GFP plasmids were kindly provided by Dr. Yule Liu (Tsinghua University, China). To generate the TRV-PoPDS construct, a 195-bp PoPDS fragment was PCRamplified using specific primers (Table 1), and cloned into the pUCm-T vector by T4 DNA

144 ligase (Sangon, China). This recombinant plasmid was digested with BamHI and EcoRI restriction enzymes, and the fragment of PoPDS (GenBank accession number: MK733916) was ligated into corresponding sites of TRV2 vector (Fig. S2). The resulting construct was then 
147 transformed into Eacherichia coli strain DH5a competent cells, which were selected on LB

148 plates containing $50 \mathrm{mg} \mathrm{l}^{-1}$ of kanamycin. PCR was used to examine the presence of PoPDS

149 insert in the generated construct.

150

151

\section{Agro-inoculation of TRV vector}

152

TRV1, TRV2, and its derivatives were introduced into Agrobacterium tumefaciens strain

153 GV3101 via freeze-thaw method (Yan et al., 2012). The transformed bacteria bearing TRV constructs were cultured in LB medium supplemented with $40 \mathrm{mg} \mathrm{l}^{-1}$ kanamycin, $20 \mathrm{mg} \mathrm{l}^{-1}$ gentamicin, $10 \mathrm{mM}$ MES, and $20 \mu \mathrm{M}$ acetosyringone at $28{ }^{\circ} \mathrm{C}$ in a growth chamber for $48 \mathrm{~h}$. Agrobacterium cultures were centrifuged at $4000 \mathrm{~g}$ for $20 \mathrm{~min}$, and resuspended in the infiltration buffer (10 $\mathrm{mM} \mathrm{MgCl}_{2}, 10 \mathrm{mM}$ MES, and $200 \mu \mathrm{M}$ acetosyringone) to a final $\mathrm{OD}_{600}$ of temperature and mixed together in a 1:1 ratio before inoculation. For syringe infiltration, the abaxial sides of two or three fully expanded leaves were injected using a 1-ml needleless syringe.

For vacuum infiltration, the whole plants were submerged in the infiltration buffer and subjected

to $0.1 \mathrm{MPa}$ vacuum pressure for $20 \mathrm{~min}$. Approximately 50 tree peony seedlings were inoculated

by vacuum method for each assay.

Semi-quantitative RT-PCR and quantitative real-time PCR

Total RNA was extracted from inoculated and systemically-infected leaves of tree peony seedlings, and purified with RNase-free DNase (Takara, Japan). First-strand cDNA as the 
168 169

170

172 173

174 175

176

177 178 179 180

181 182 183 184 185 186 188

187 A GFP-specific antibody (Abcam Inc) was used to implement western blot analysis. Proteins

template for PCR was synthesized from 2-5 $\mu \mathrm{g}$ of total RNA. Three primer pairs were designed to detect the presence of TRV (Table 1). Since the forward and reverse primers of TRV2-2 covered the multiple cloning sites (MCS), the size of resulting product varied depending on the inserts in the site, whereas the TRV1 and TRV2-1 primers led to the bands with the same sizes (Sun et al., 2016). The PCR products were analyzed through electrophoresis using a Molecular Imager Gel Doc XR+ System (Bio-Rad, USA). Quantitative real-time PCR (qRT-PCR) was carried out using SYBR Premix Ex Taq II (Takara, Japan) in a $20-\mu 1$ PCR mixture and analyzed by a StepOnePlus Real-time PCR System (Applied Biosystems, USA). 18S-26S internal transcribed spacer was used as an internal control to normalize the expression data (Zhang et al., 2018). The PCR primers, used for the determination of transcript abundances of PoPDS, were designed outside the region of the inserted fragment to avoid amplification of the fragment included in TRV2 construct.

\section{GFP imaging}

Transient assay of GFP in the inoculated leaf and root cells of $P$. ostii was conducted based on the agro-infiltration with TRV-GFP. GFP fluorescence was detected and photographed using a laser scanning confocal microscope (Leica TCS SP8).

\section{Western blot} were extracted from leaves and roots of $P$. ostii plants, with $300 \mu \mathrm{L}$ extraction buffer $(100 \mathrm{mM}$ 
189 Tris $\mathrm{pH}=6.8,2.5 \%$ SDS, $100 \mathrm{mM}$ dithiothreitol, $100 \mathrm{mM} \mathrm{NaCl}$, and $10 \%$ glycerol). Bradford 190 assay was used to determine protein quantities, and equal amounts of proteins for each sample 191 were separated by 10\% SDS-PAGE (Bradford, 1976). Next, proteins were transferred to a 192 polyvinylidene difluoride membrane (GE healthcare). CP-GFP was detected after an overnight 193 incubation at room temperature with a 1:10,000 dilution of the anti-GFP antibody conjugated to 194 alkaline phosphatase (Tian et al., 2014). Alkaline phosphatase was detected using a 195 chemiluminescent substrate (CSPD; Roche) and exposed to X-ray film (Kodak X-OMAT BT 196 197 198 199 200 201 202 203 204 205 206 207 208 209

\section{Results}

\section{Comparison of the agro-infiltration methods}

In view of the woody characteristics of tree peony, choosing a plant with optimal age and size for VIGS assay is pre-requisite. Three-year-old young plantlets were therefore used because of their delicate underground roots, small plant type, and high occurrence of new leaves (Fig. 1a). To determine the most appropriate method of Agrobacterium-mediated TRV infection in tree peony, leaf syringe-infiltration and seedling vacuum-infiltration were selected for comparison (Fig. 1b).

We found that the vacuum infiltration brought about a more sufficient permeation of bacterial cultures through the abaxial leaf surface than the syringe infiltration, which made the infiltration happen only at the inoculation sites. Furthermore, the syringe infiltration inevitably caused obvious mechanical damage to leaf tissues (Fig. 1c). Semi-quantitative RT-PCR analysis indicated that TRV1 and TRV2 transcripts were detected in all inoculated leaves by both 
210 infiltration methods, but not in untreated leaves (Fig. 1d). And, the TRV transcripts accumulation

211 levels in vacuum-infiltrated leaves are obviously higher than that in syringe-infiltrated leaves.

212 According to the results, vacuum infiltration was used for subsequent gene-silencing experiments.

Identification of PoPDS

Phytoene desaturase $(P D S)$ is commonly used as a visible reporter for silencing. Based on the transcriptome data obtained from developing leaves of tree peony, we PCR-amplified the open

217 reading frame (ORF) nucleotide sequence of $P$. ostii $P D S$, annotated as PoPDS. PoPDS was predicted to encode a protein of 575 amino acids, and conserved domain analysis revealed a

putative dinucleotide binding domain in its deduced protein sequence. Multiple sequence alignments showed that amino acid sequence of PoPDS shared high similarity with the homologies from other plant species, such as Vitis vinifera, Nicotiana tabacum, Arabidopsis thaliana, and Petunia hybrida (Fig. S2). The full-length amino acid sequence of PoPDS had $83.3 \%, 82.09 \%, 79.96 \%$, and $80.7 \%$ identities with those of four plant species, respectively.

Silencing of PoPDS in P. ostii leaves

To assess the feasibility of TRV-based VIGS in tree peony, we introduced a 195-bp conserved fragment of PoPDS into TRV2 vector, and generated a TRV-PoPDS recombinant (Fig. 2). Upon Agrobacterium-mediated infection, similar necrotic symptoms occurred in the edge of leaves infiltrated with TRV empty vector and TRV-PoPDS, while the remaining area seems normal 
231 the first newly developed leaves at 4 weeks post inoculation. White spots or sectors were clearly

232 observed throughout the upper leaves particularly around leaf main veins. This phenotype

233 remained stable and persisted for about 2 months under growth chamber conditions (Fig. S3).

234 To confirm the correlation of leaf photobleaching with the presence of the viral vectors, TRV

235 accumulation was examined using semi-quantitative RT-PCR. TRV1 and TRV2 were detected in

236 TRV empty vector- and TRV-PoPDS-infected leaves, but not in the mock control plants (Fig.

237 3b). When using primers covering MCS of TRV2 vector, a fragment carrying PoPDS insert was

238 detected in the leaves agro-infiltrated with TRV-PoPDS. 18S-26S internal transcribed spacer was

239 referred as an internal control for normalization of gene expression. QRT-PCR analysis

240 demonstrated that transcript abundances of PoPDS were significantly reduced in photobleached

241 leaves of plants infiltrated with TRV-PoPDS, compared with that in mock- and TRV empty

242 vector-inoculated seedlings (Fig. 3c). These results suggested that the leaf photobleaching

243 phenotype was initiated by PoPDS silencing. It indicated that the PoPDS of tree peony could be

244 silenced by VIGS and TRV infection was systemically established.

\section{Validation of TRV-GFP in $P$. ostii leaves and roots}

Apart from TRV-PoPDS, another visualizable vector TRV-GFP, in which the EGFP coding sequence was fused to coat protein ORF of TRV2, was used for infiltration to monitor virus spread in P. ostii. Under a confocal microscope, GFP fluorescence was observed in the newly emerging leaves and roots of plants at 5 days post inoculation (dpi) with TRV-GFP, indicating the capability of TRV vector to express foreign genes in different tree peony tissues. No 
252 fluorescence signals were detected in mock control leaves and roots (Fig. 4).

253 Moreover, we performed western blot analysis to check the expression of GFP protein in 254 infected leaves and roots. As illustrated in Fig. 5a, GFP proteins were accumulated in the leaves 255 and roots of plants inoculated with TRV-GFP, whereas no GFP bands were found in control 256 plants. By contrast, the GFP abundances in infected roots appeared to be much higher than that 257 in infected leaves (Fig. 5a). Semi-quantitative RT-PCR analysis revealed a consistent variance 258 that the roots exhibited more transcripts of TRV1, TRV2, and GFP than the leaves (Fig. 5b). The 259 data suggested that the systemic movement of TRV vector in tree peony plants could be effectively supervised via the GFP-tagged expression.

Discussion

In addition to the significance floral characteristics of tree peony, its roots contains some special secondary metabolites, which are generally used as traditional Chinese medical materials, and its leaves also has excellent ornamental values owing to its changeable color during the early growth period (Luo et al., 2017; Li et al., 2018). Therefore, there are considerable interests in evaluating the gene function in both roots and leaves of tree peony. However, an effective genetic transformation system is still unavailable in tree peony because of severe callus browning and tough plant regeneration (Liu \& Jia, 2010). Few studies on molecular functional identification have been performed in tree peony due to this limitation. It seems likely that a needed. 

efficient tool for functional assessment of genes (Wege et al., 2007; Velásquez et al., 2009). In the present study, whether TRV-based vector could be used for silencing endogenous genes in tree peony was investigated. Our results demonstrated that the conventional leaf syringe-

277 infiltration method is laborious and it resulted in an inadequate infiltration to $P$. ostii leaves, 278 when compared with seedling vacuum-infiltration. It is quite likely that the physiological structure of tree peony leaf affected the entering of agrobacterial mixture. Not many stomatal apparatus existed in the lower epidermis of tree peony young leaf, and its leaf

a limited area of leaf could be effectively infiltrated with TRV constructs via syringe injection.

Additionally, the thin tree peony leaves were prone to suffer mechanical damage from syringeinfiltration method. Previous studies also showed that the vacuum approach was more effective than other infiltration methods in woody plants (Ye et al., 2009; Liu et al., 2014). Thus, a vacuum-infiltration into the whole plant is probably considered as a good choice, when it comes to species that are difficult to infect.

Concerning the experimental materials for inoculation, it is well known that tree peony has a long juvenile stage that commonly lasts for about 3 years, during which the root is the main growing part (Wang et al., 2015). This development feature confined the application of VIGS on tree peony plants. Three-year-old seedlings of tree peony were consequently selected as agroinoculated objects in our work. Since the plants at this stage were favorable to vacuum 
294

295

296

297

298

299

300

301

302

303

304

305

306

307

308

309

310

311

312

313

314

phenotype of marker gene, such as $P D S$-silenced leaf photobleaching or chalcone synthase (CHS)silenced white-corollas phenotypes, requires upspring of systemically-infected tissues. Our results proved that a significant gene silencing took place in newly-developed leaves of triennial P. ostii plants. Because no reproductive buds were formed at this stage, a trial of gene silencing in tree peony floral organs via VIGS will be made in future work.

$P D S$ has been frequently used as an indicator gene in VIGS systems because the silencing of $P D S$ reduces photoprotective carotenoid levels in green tissues and thereby leads to chlorophyll photooxidation and tissue bleaching (Kumagai et al., 1995). In this study, we cloned the PDS gene from $P$. ostii leaves and constructed the TRV-PoPDS vector to unravel the function of PoPDS and verify the possibility of applying VIGS in tree peony. After infiltration with TRVPoPDS, an expected silencing phynotype (photobleaching) was observed in systemicallyinfected leaves, while the directly inoculated leaves showed lesions resembling those of TRV empty vector. The results mentioned above indicated that a systemic TRV viral infection was established, and it was essential for the VIGS application. The silencing of PoPDS also demonstrated that TRV-based VIGS could be used as an effective method towards functional characterization of genes in tree peony plants.

It is noteworthy that almost all photobleached leaves resulting from TRV-PoPDS infection exhibited variegated phenotypes as white spots or sectors not completely white (Fig. 3a), and we hypothesized that multiple factors may contribute to it. The post-inoculation growth temperature largely influences the efficiency of VIGS-based gene silencing. It has been reported that low temperature enhances gene silencing efficiency when TRV-mediated VIGS is employed in 
315 tomato (Fu et al., 2006). But a conflicting finding is that low temperature suppresses gene

316 silencing through the prevention of siRNA formation in N. benthamiana (Szittya et al., 2003).

317 The length of inserted fragment in viral vector is also closely associated with gene silencing

318 efficiency. As reported previously, different lengths of $P D S$ inserts result in varied

319 photobleaching patterns and ranges in TRV-infected tobacco (Liu \& Page, 2008; Ye et al., 2009).

320 Our VIGS procedure hence requires further optimization in temperature and inserted fragment

321 size in future work. Furthermore, the PoPDS-silenced phenotypes were particularly significant

322 along the leaf vein (Fig. 3a). It is in agreement with the results that viral propagation and/or

323 systemic silencing response occur mainly along the vascular bundle system (Wege et al., 2007).

324 In order to visualize viral accumulation in infiltrated tree peony plants, the TRV-GFP vector

was used. The GFP, a fluorescent protein from jellyfish (Aequorea victoria), does not participate

in biological processes of plants. The gene was overexpressed driven by the $35 \mathrm{~S}$ promoter and

used as a marker to trace the presence of virus (Tian et al., 2014). In the present work, green

fluorescence was observed in the roots and leaves of infected tree peony seedlings at 5 dpi, and

the accumulation levels of TRV1, TRV2, and GFP were also detected (Fig. 4). The concentration

of GFP protein was able to reflect the viral load and degree of silencing (Tian et al., 2014).

Previous findings proved that TRV virus possesses the ability to move efficiently within the

roots of infected plants (Macfarlane \& Popovich, 2000). We found a higher transcript and

protein levels of GFP in roots than that in leaves. It is concluded that virus infection may happen

mainly in roots at first and then spread into newly-developed leaves after vacuum infiltration.

Future work will examine the underlying mechanism for the discrepancy of TRV replication and 
336 movement in roots and leaves of tree peony. Altogether, it suggested that the TRV-GFP vector

337 was available to tree peony plants and suitable for monitoring the systemic spread of TRV

338 carrying target gene fragments. An advantage is that the employment of TRV-GFP construct

339 could avoid the destruction of the photosynthetic apparatus caused by $P D S$-silenced leaf

340 photobleaching.

341

342 Conclusion

343 In conclusion, our results indicated that an effective TRV-based VIGS system was established in

$344 P$. ostii based on TRV-PoPDS and TRV-GFP constructs. Seedling vacuum-infiltration was

345 determined as an appropriate method for Agrobacterium-mediated infection of TRV, compared

346 with leaf syringe-infiltration. A remarkable photobleaching phenotype was observed in TRV-

347 PoPDS-infected upper new leaves, which was concomitant with substantial reduction in PoPDS

348 transcripts. The detection of GFP fluorescence and accumulation levels in leaves and roots

349 infected with TRV-GFP revealed TRV is a versatile tool to analyze gene function in different

350 tissues of tree peony. Thus, this system we developed will be greatly helpful to characterize the

351 function of genes associated with various molecular and physiological processes in tree peony.

352

353 Acknowledgements

354 We thank Xiang Li and Xiaotong Ji for experimental assistance in agro-infiltration with syringe and vacuum. We are grateful to Yule Liu's favor for kindly providing TRV-GFP vector. 
References

Bartel DP. 2004. MicroRNAs: genomics, biogenesis, mechanism, and function. Cell 116:281297

Baulcombe D. 1999. Viruses and gene silencing in plants. Arch Virol Suppl 15:189-201

Bradford M. 1976. A rapid and sensitive method for quantitation of microgram quantities of protein utilizing the principle of proteindye binding. Analytical Biochemistry 72:248-254

Burch-Smith T, Anderson J, Martin GK, Sp. 2004. Applications and advantages of virusinduced gene silencing for gene function studies in plants. Plant Journal 39:734-746

Burchsmith TM, Schiff M, Liu Y, Dineshkumar SP. 2006. Efficient virus-induced gene silencing in Arabidopsis. Plant Physiology 142:21-27

Cheng F, Li J, and Yu L. 1998. Exportation of Chinese Tree Peonies (Mudan) and their developments in other countries.II.Wild species. Estuarine Coastal \& Shelf Science 73:223-235.

Cunningham FX, and Gantt E. 1998. GENES AND ENZYMES OF CAROTENOID BIOSYNTHESIS IN PLANTS. Annual Review of Plant Biology 49:557-583.

Dinesh-Kumar SP, Anandalakshmi R, Marathe R, Schiff M, and Liu Y. 2011. Virusinduced gene silencing. Methods Mol Biol 236:287-294.

Faivrerampant O, Gilroy EM, Hrubikova K, Hein I, Millam S, Loake GJ, Birch PRJ, Taylor MA, and Lacomme C. 2004. Potato Virus X-Induced Gene Silencing in Leaves and Tubers of Potato. Plant Physiology 134:1308-1316.

Fu DQ, Zhu BZ, Zhu HL, Zhang HX, Xie YH, Jiang WB, Zhao XD, and Luo KB. 2006. Enhancement of virus-induced gene silencing in tomato by low temperature and low humidity. Molecules \& Cells 21:153-160.

Holzberg S, Brosio P, Gross CS, and Pogue GP. 2002. Barley stripe mosaic virus - induced gene silencing in a monocot plant. Plant Journal 30:315-327.

Jia HF, Chai YM, Li CL, Lu D, Luo JJ, Qin L, and Shen YY. 2011. Abscisic acid plays an important role in the regulation of strawberry fruit ripening. Plant Physiology 157:188.

Kumagai MH, Donson J, Dellacioppa GR, Harvey D, Hanley KM, and Grill LK. 1995. Cytoplasmic inhibition of carotenoid biosynthesis with virus-derived RNA. Proceedings of the National Academy of Sciences of the United States of America 92:1679-1683.

Kurth EG, Peremyslov VV, Prokhnevsky AI, Kasschau KD, Miller M, Carrington JC, and Dolja VV. 2012. Virus-Derived Gene Expression and RNA Interference Vector for Grapevine. Journal of Virology 86:6002-6009.

Li C, Hui DU, and Wang L. 2009. Flavonoid Composition and Antioxidant Activity of Tree Peony (Paeonia Section Moutan) Yellow Flowers. Journal of Agricultural \& Food Chemistry 57:8496-8503.

Li J, Han J, Hu Y, and Yang J. 2016. Selection of Reference Genes for Quantitative Real-Time PCR during Flower Development in Tree Peony (Paeonia suffruticosa Andr.). Front Plant Sci 7:516. 
Li S, Wu Q, Yin D, Feng C, Liu Z, and Wang L. 2018. Phytochemical variation among the traditional Chinese medicine $\mathrm{Mu}$ Dan $\mathrm{Pi}$ from Paeonia suffruticosa (tree peony). Phytochemistry 146:16-24.

Liu E, and Page JE. 2008. Optimized cDNA libraries for virus-induced gene silencing (VIGS) using tobacco rattle virus. Plant Methods 4:5-5.

Liu HC, and Jia WQ. 2010. Establishment of plantlet regeneration system of tree peony through lateral buds cutting and carving. Acta Horticulturae Sinica 37:1471-1476.

Liu Y, Schiff M, and Dineshkumar SP. 2002. Virus - induced gene silencing in tomato. Plant Journal 31:777-786.

Liu Y, Wei S, Zeng S, Huang W, Di L, Hu W, Shen X, and Ying W. 2014. Virus-induced gene silencing in two novel functional plants, Lycium barbarum L. and Lycium ruthenicum Murr. Scientia Horticulturae 170:267-274.

Luo J, Shi Q, Niu L, and Zhang Y. 2017. Transcriptomic Analysis of Leaf in Tree Peony Reveals Differentially Expressed Pigments Genes. Molecules 22:324.

Macfarlane SA, and Popovich AH. 2000. Efficient expression of foreign proteins in roots from tobravirus vectors. Virology 267:29-35.

Purkayastha A, and Dasgupta I. 2009. Virus-induced gene silencing: a versatile tool for discovery of gene functions in plants. Plant Physiology and Biochemistry 47:967-976.

Ratcliff F, ., Martin-Hernandez AM, and Baulcombe DC. 2001. Technical Advance. Tobacco rattle virus as a vector for analysis of gene function by silencing. Plant Journal 25:237-245.

Reid M, Chen JC, and Jiang CZ. 2009. Virus-Induced Gene Silencing for Functional Characterization of Genes in Petunia. In: Gerats T, Strommer J, eds. Petunia. Berlin Heidelberg: Springer, 381-394.

Robertson NL, Brown KL, Winton LM, and Holloway PS. 2009. First report of Tobacco rattle virus in Peony in Alaska. Plant Disease 93:675-675.

Ruiz MT, Voinnet O, and Baulcombe DC. 1998. Initiation and Maintenance of Virus-Induced Gene Silencing. The Plant Cell 10:937-946.

Senthilkumar M, and Mysore KS. 2011. New dimensions for VIGS in plant functional genomics. Trends in Plant Science 16:656-665.

Singh A, Kumar P, Jiang CZ, and Reid MS. 2013. TRV Based Virus Induced Gene Silencing in Gladiolus ( Gladiolus grandiflorus L.), A Monocotyledonous Ornamental Plant. International Journal of Plant Research 26:170.

Stilio VS, Di, Kumar RA, Oddone AM, Tolkin TR, Patricia S, and Kacie MC. 2010. Virusinduced gene silencing as a tool for comparative functional studies in Thalictrum. Plos One 5:e12064.

Sun D, Li S, Niu L, Reid MS, Zhang Y, and Jiang C. 2017. PhOBF1, a petunia ocs element binding factor, plays an important role in antiviral RNA silencing. Journal of Experimental Botany 68:915-930. 
434

435

436

437

438

439

440

441

442

443

444

445

446

447

448

449

450

451

452

453

454

455

456

457

458

459

460

461

462

463

464

465

466

467

468

469

470

471

472

473

Sun D, Nandety RS, Zhang Y, Reid MS, Niu L, and Jiang C. 2016. A petunia ethyleneresponsive element binding factor, PhERF2, plays an important role in antiviral RNA silencing. Journal of Experimental Botany 67:3353-3365.

Szittya G, Silhavy D, Molnar A, Havelda Z, Lovas A, Lakatos L, Banfalvi Z, and Burgyan J. 2003. Low temperature inhibits RNA silencing-mediated defence by the control of siRNA generation. The EMBO Journal 22:633-640.

Tasaki K, Terada H, Masuta C, and Yamagishi M. 2016. Virus-induced gene silencing (VIGS) in Lilium leichtlinii using the Cucumber mosaic virus vector. Plant Biotechnology 33:373381.

Tian J, Pei H, Zhang S, Chen J, Chen W, Yang R, Meng Y, You J, Gao J, and Ma N. 2014. TRV-GFP: a modified Tobacco rattle virus vector for efficient and visualizable analysis of gene function. Journal of Experimental Botany 65:311-322.

Velásquez AC, Chakravarthy S, and Martin GB. 2009. Virus-induced gene silencing (VIGS) in Nicotiana benthamiana and tomato. Journal of Visualized Experiments 28: 1292.

Wang S, Beruto M, Xue J, Zhu F, Liu C, Yan Y, and Zhang X. 2015. Molecular cloning and potential function prediction of homologous SOC1 genes in tree peony. Plant Cell Reports 34:1459-1471.

Wege S, Scholz A, Gleissberg S, and Becker A. 2007. Highly Efficient Virus-induced Gene Silencing (VIGS) in California Poppy (Eschscholzia californica): An Evaluation of VIGS as a Strategy to Obtain Functional Data from Non-model Plants. Annals of Botany 100:641649.

Wu J, Cai C, Cheng F, Cui H, and Zhou H. 2014. Characterisation and development of ESTSSR markers in tree peony using transcriptome sequences. Molecular Breeding 34:18531866.

Wu L, Ma N, Jia Y, Zhang Y, Feng M, Jiang CZ, Ma C, and Gao J. 2016. An ethyleneinduced regulatory module delays flower senescence by regulating cytokinin content. Plant Physiology 173: 853-862.

Yamagishi N, and Yoshikawa N. 2013. Highly Efficient Virus-Induced Gene Silencing in Apple and Soybean by Apple Latent Spherical Virus Vector and Biolistic Inoculation. Methods of Molecular Biology 975:167-181.

Yan H, Fu D, Zhu B, Liu H, Shen X, and Luo Y. 2012. Sprout vacuum-infiltration: a simple and efficient agroinoculation method for virus-induced gene silencing in diverse solanaceous species. Plant Cell Reports 31:1713-1722.

Ye J, Qu J, Bui HTN, and Chua N. 2009. Rapid analysis of Jatropha curcas gene functions by virus - induced gene silencing. Plant Biotechnology Journal 7:964-976.

Yin DD, Xu WZ, Shu QY, Li SS, Wu Q, Feng CY, Gu ZY, and Wang LS. 2018. Fatty acid desaturase 3 ( PsFAD3 ) from Paeonia suffruticosa reveals high $\alpha$-linolenic acid accumulation. Plant Science 274:212-222.

Zhang J, Yu D, Zhang Y, Liu K, Xu K, Zhang F, Wang J, Tan G, Nie X, and Ji Q. 2017. Vacuum and Co-cultivation Agroinfiltration of (Germinated) Seeds Results in Tobacco 
474 Rattle Virus (TRV) Mediated Whole-Plant Virus-Induced Gene Silencing (VIGS) in Wheat $475 \quad$ and Maize. Frontiers in Plant Science 8:393.

476 Zhang Q, Yu R, Xie L, Rahman M, Kilaru A, Niu L, and Zhang Y. 2018. Fatty Acid and 477 Associated Gene Expression Analyses of Three Tree Peony Species Reveal Key Genes for $478 \alpha$-Linolenic Acid Synthesis in Seeds. Frontiers in Plant Science 9:106.

Zhang Y, Cheng Y, Ya H, Xu S, and Han J. 2015a. Transcriptome sequencing of purple petal spot region in tree peony reveals differentially expressed anthocyanin structural genes. Frontiers in Plant Science 6:964.

Zhang Y, Zhang L, Gai S, Liu C, and Lu S. 2015b. Cloning and expression analysis of the R2R3-PsMYB1 gene associated with bud dormancy during chilling treatment in the tree peony (Paeonia suffruticosa). Plant Growth Regulation 75:667-676. 


\section{Table 1 (on next page)}

Primers used for RT-PCR amplification and construction of recombinant TRV2 plasmids 
1 Table 1 Primers used for RT-PCR amplification and construction of recombinant TRV2 2 plasmids.

\begin{tabular}{|c|c|c|c|}
\hline Primer name & Nucleotide sequence $\left(5^{\prime}-3^{\prime}\right)$ & Purpose & Size \\
\hline PoPDS-F1 & TCGGAGTTGGGTTCGCTGC & \multirow{2}{*}{$\begin{array}{l}\text { Cloning of } P o P D S \\
\text { coding region }\end{array}$} & \multirow{2}{*}{$1797 \mathrm{bp}$} \\
\hline PoPDS-R1 & ATTCTGATGTGTTTTGTAGCC & & \\
\hline PoPDS-F2 & CAGCCGATTTGATTTCCTTG & \multirow{2}{*}{$\begin{array}{l}\text { Cloning of inserted } \\
\text { fragment for VIGS }\end{array}$} & \multirow{2}{*}{$195 \mathrm{bp}$} \\
\hline PoPDS-R2 & CCTTGTTTTCTCATCCAGTC & & \\
\hline PoPDS-F3 & AGTCATTGGGGGGTCAGGTCCG & \multirow{2}{*}{ RT-PCR } & \multirow{2}{*}{$312 b p$} \\
\hline PoPDS-R3 & CAGCATACACACTCAGAAGGGG & & \\
\hline TRV1-F & CAGTCTATACACAGAAACAGA & \multirow{2}{*}{$\begin{array}{l}\text { TRV1-RNA } \\
\text { detection }\end{array}$} & \multirow{2}{*}{$463 \mathrm{bp}$} \\
\hline TRV1-R & GACGTGTGTACTCAAGGGTT & & \\
\hline TRV2-1F & GGCTAACAGTGCTCTTGGTG & \multirow{2}{*}{$\begin{array}{l}\text { TRV2-RNA } \\
\text { detection }\end{array}$} & \multirow{2}{*}{$359 \mathrm{bp}$} \\
\hline TRV2-1R & GTATCGGACCTCCACTCGC & & \\
\hline TRV2-2F & CGAGTGGAGGTCCGATACG & \multirow{2}{*}{$\begin{array}{l}\text { TRV2-RNA } \\
\text { (containing inserted } \\
\text { fragment) detection }\end{array}$} & \multirow{2}{*}{$\begin{array}{l}\text { Depending } \\
\text { on insert }\end{array}$} \\
\hline TRV2-2R & CGGTTCATGGATTCGGTTAG & & \\
\hline GFP-F & ATGGCCAACACTTGTCACTACTT & \multirow{2}{*}{ GFP-RNA detection } & \multirow{2}{*}{$260 \mathrm{bp}$} \\
\hline GFP-R & ATTCCAATTTGTGTCCAAGAATG & & \\
\hline 18S-26S-ITS-F & ACCGTTGATTCGCACAATTGGTCA & \multirow{2}{*}{ RT-PCR } & \multirow{2}{*}{$150 \mathrm{bp}$} \\
\hline 18S-26S-ITS-R & TACTGCGGGTCGGCAATCGGACG & & \\
\hline
\end{tabular}

3

4 


\section{Figure 1}

Comparison of syringe-infiltration and vacuum-infiltration methods with the TRV empty vector

a Three-year-old $P$. ostii plantlets at 4 weeks post germination used for agro-infiltration. b

Schematic depiction of Agrobacterium-mediated TRV inoculation in P. ostii plants using

syringe and vacuum methods. c The $P$. ostii leaves subjected to syringe- and vacuuminfiltration with TRV empty vector. $\mathbf{d}$ Semi-quantitative RT-PCR analysis of TRV1 and TRV2-1 accumulation levels in TRV empty vector-inoculated leaves by syringe and vacuum methods. 18S-26S internal transcribed spacer (18S-26S ITS) was used as internal standard.

a

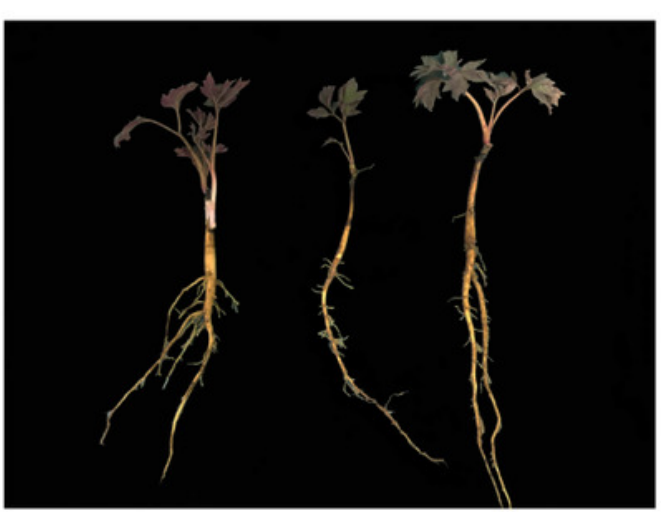

b

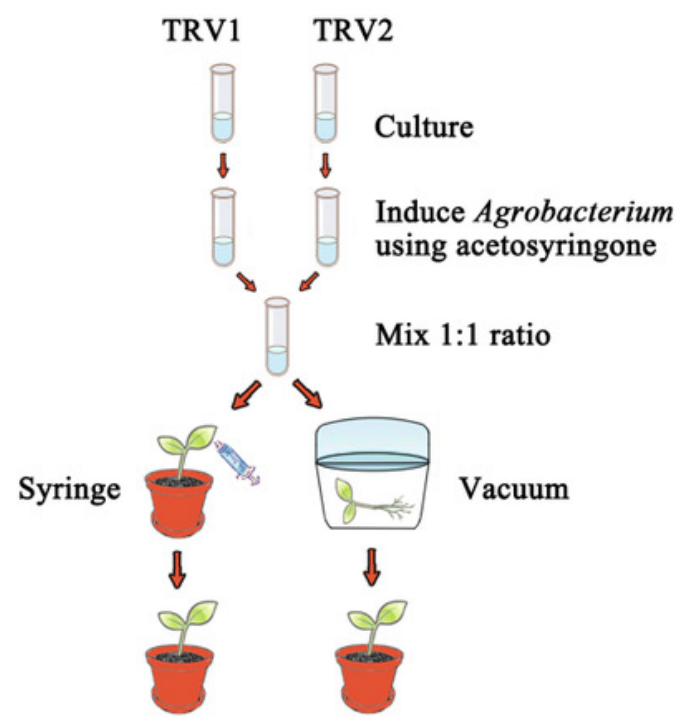

c
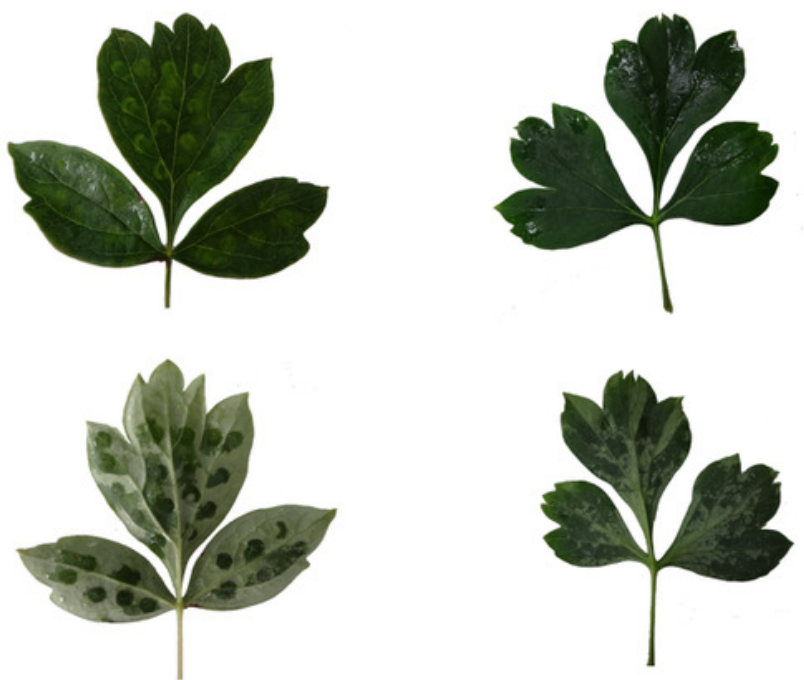

Syringe-infiltration

Vacuum-infiltration

d

Untreated Syringe-infiltration Vacuum-infiltration

TRV1

TRV2-1

18S-26S-ITS 


\section{Figure 2}

Schematic representation of TRV constructs used in this study

a The CDNA of POPDS insert for its introduction into TRV vector. PoPDS-F1/PoPDS-R1 was used to amplify the open reading frame region of PoPDS, PoPDS-F2/PoPDS-R2 targeted the inserted fragment of POPDS (the black box), and PoPDS-F3/PoPDS-R3 was designed for quantitative real-time PCR. $\mathbf{b}$ The structures of TRV1, TRV2, TRV2-PoPDS, and TRV2-GFP. The arrows indicate the different primer pairs for examining TRV1, TRV2-1, TRV2-2, and GFP transcript levels. LB left border, RB right border, MP movement protein, 16K $16 \mathrm{Kd}$ protein, Rz self-cleaving ribozyme, NOSt NOS terminator, CP coat protein, MCS multiple cloning site. 
a

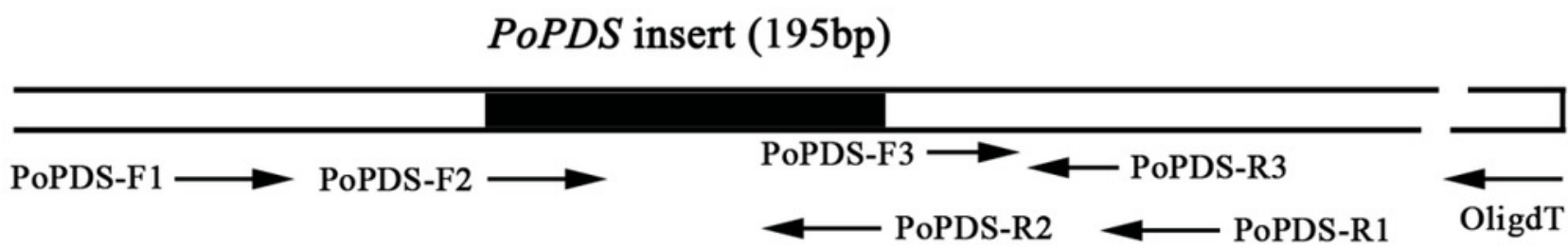

b

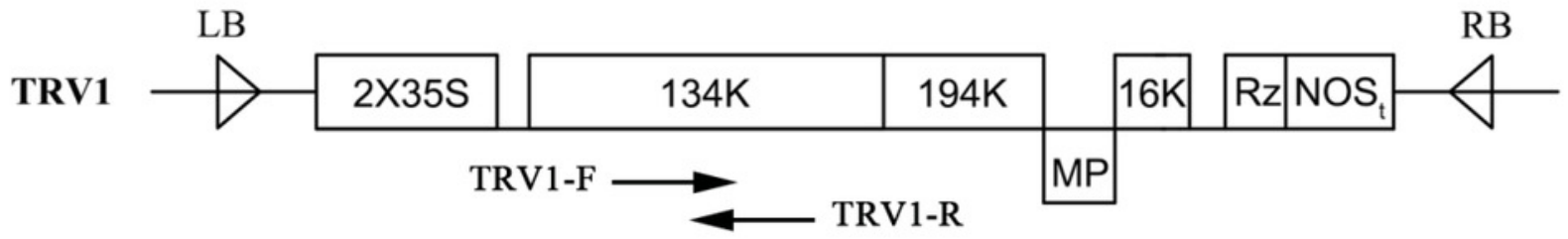

TRV2
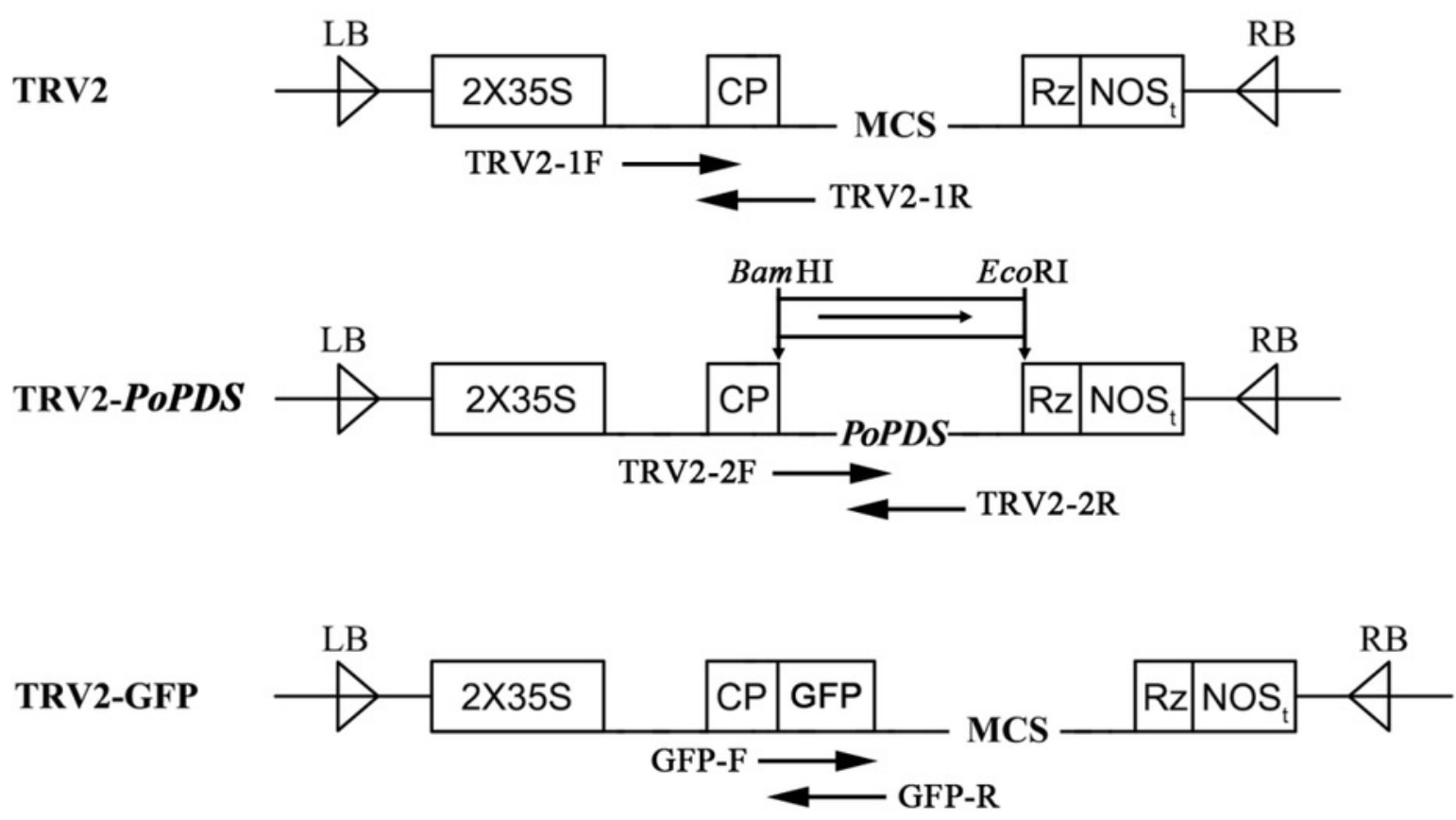


\section{Figure 3}

Silencing of POPDS in P. ostii leaves infected with TRV-PoPDS

a Representative phenotypes of mock treated, TRV- (empty vector), and TRV-PoPDS-infected leaves in P. ostii seedlings. Photobleaching phenotypes were observed in the first newlydeveloped leaves of seedlings at 5 weeks post infiltration with TRV-PoPDS. b Semiquantitative RT-PCR analysis of TRV1 and TRV2 accumulation levels in agro-infected $P$. ostii leaves.c Quantitative real-time PCR analysis of PoPDS in agro-infected $P$. ostii leaves. 18S-26S internal transcribed spacer (18S-26S ITS) was used to normalize the transcript levels, and relative expression values were calculated compared with the highest expression value taken as 1.0 (untreated). Error bars represent \pm SE of data from three independent experiments. The different letters indicate significant differences using Duncan's multiple range test at $p<0.05$. 
$\mathbf{a}$
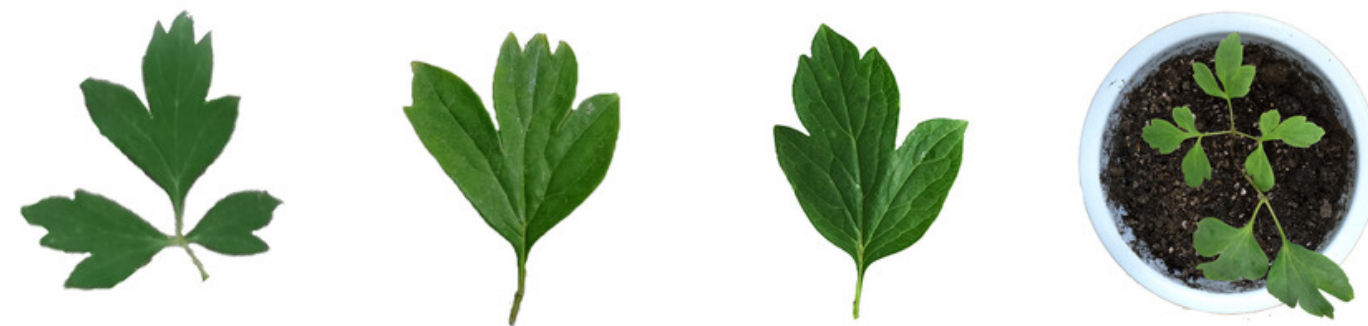

Mock
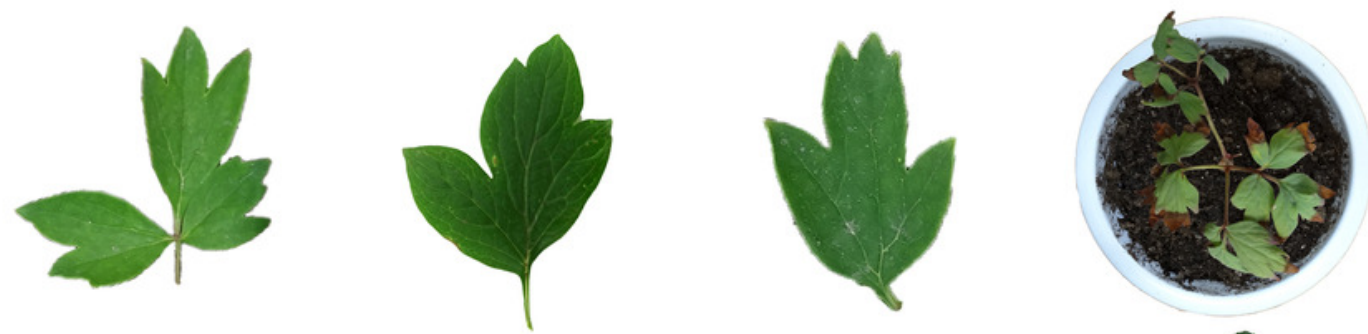

TRV
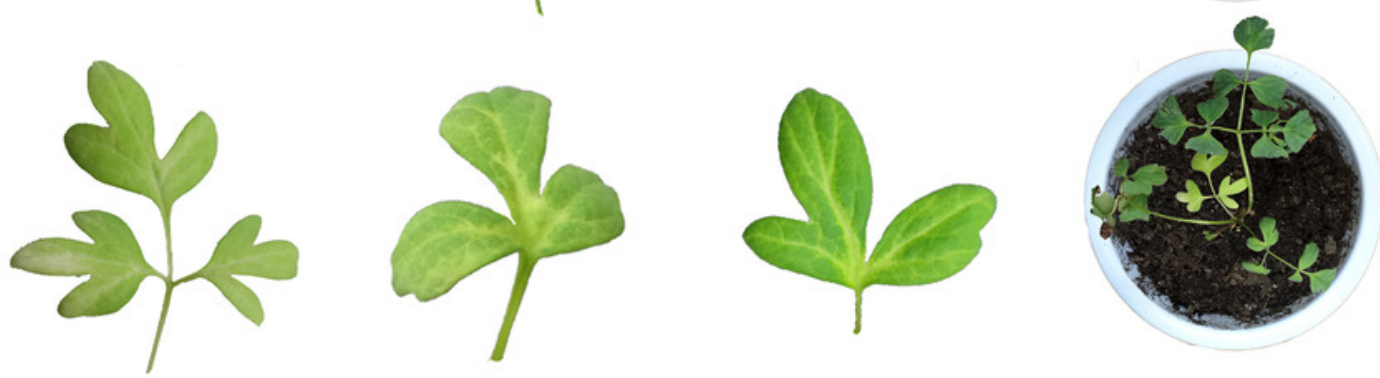

b Mock

TRV

TRV-PoPDS

TRV1

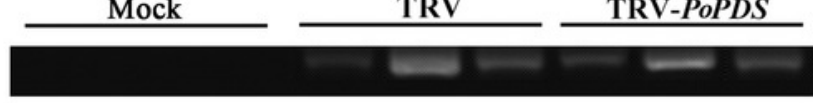

TRV2-1

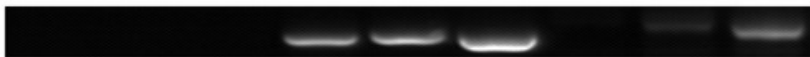

TRV2-2

18S-26S-ITS

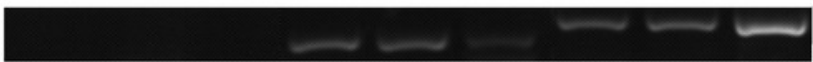

C

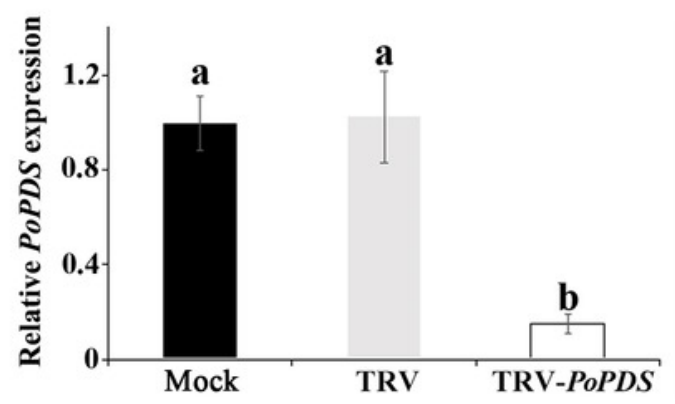




\section{Figure 4}

GFP expression in $P$. ostii leaves and roots inoculated with TRV-GFP

Confocal microscopy image of P. ostii leaves and roots infected with TRV-GFP at 5 days postinfiltration (dpi). Fluorescence was not observed in leaves $(a-c)$ and roots ( $g-i)$ of mocktreated plants. The bright field $(a, d, g, j)$, the GFP channel $(b, e, h, k)$, and the merged images $(c, f, i, I)$ of the bright field and the GFP channel are shown. Scale bars equal to 100 $\mu \mathrm{m}(\mathrm{a}-\mathrm{f})$ or $75 \mu \mathrm{m}(\mathrm{g}-\mathrm{I})$. 


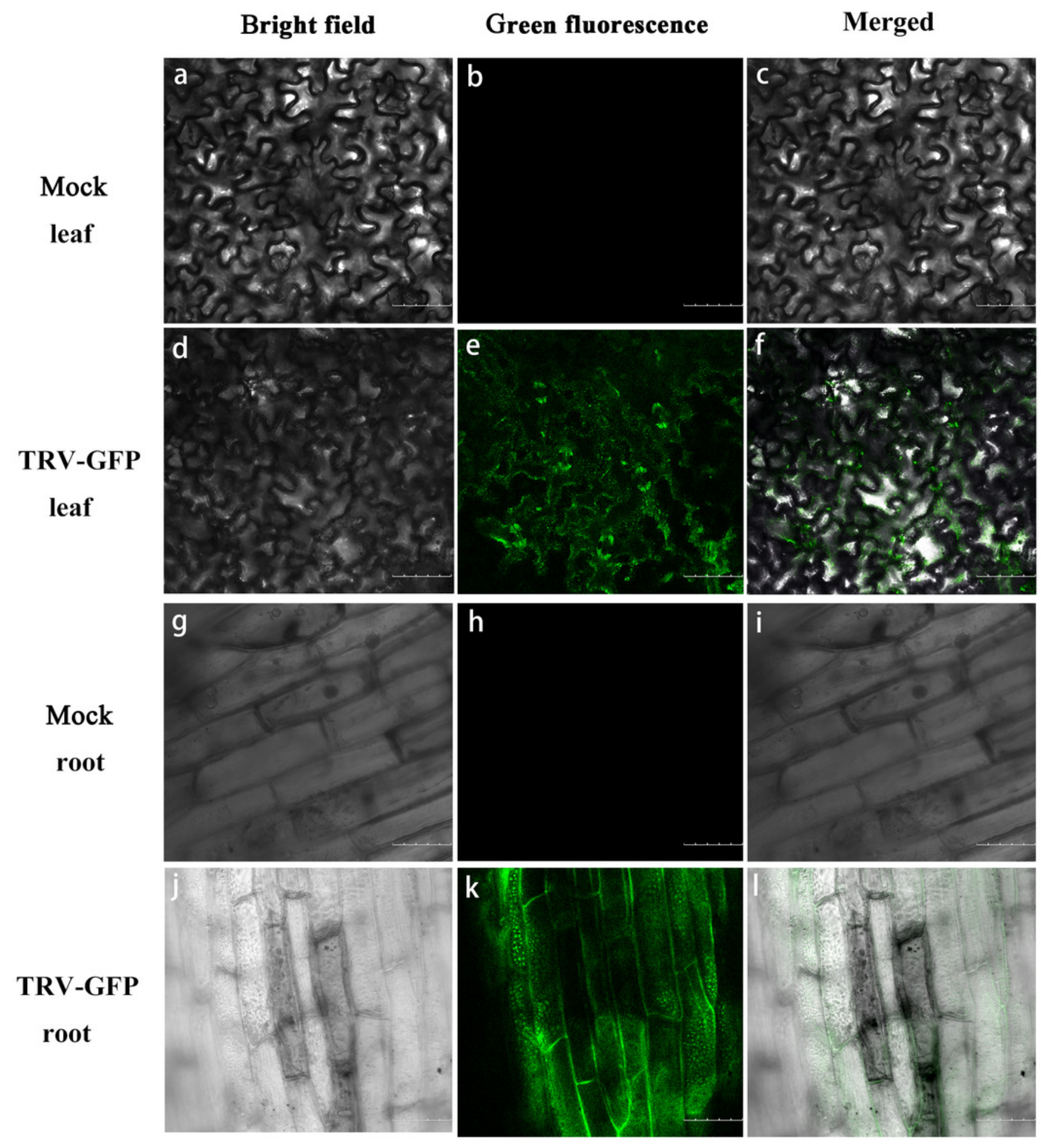




\section{Figure 5}

Detection of GFP protein accumulation in TRV-GFP-inoculated $P$. ostii leaves and roots

a Western blot analysis of CP-GFP protein levels in mock treated, TRV-GFP-infected P. ostii leaves and roots at 5 days post inoculation. Ten micrograms of protein were loaded into each lane and an anti-GFP antibody was used to detect the CP-GFP fusion protein. Coomassie blue staining was used to confirm equal loading in each lane. b Semi-quantitative RT-PCR analysis of TRV1, TRV2, and GFP accumulation levels in mock treated, TRV-GFP-infected $P$. ostii leaves and roots. 18S-26S internal transcribed spacer (18S-26S-ITS) was used as internal control. 
a

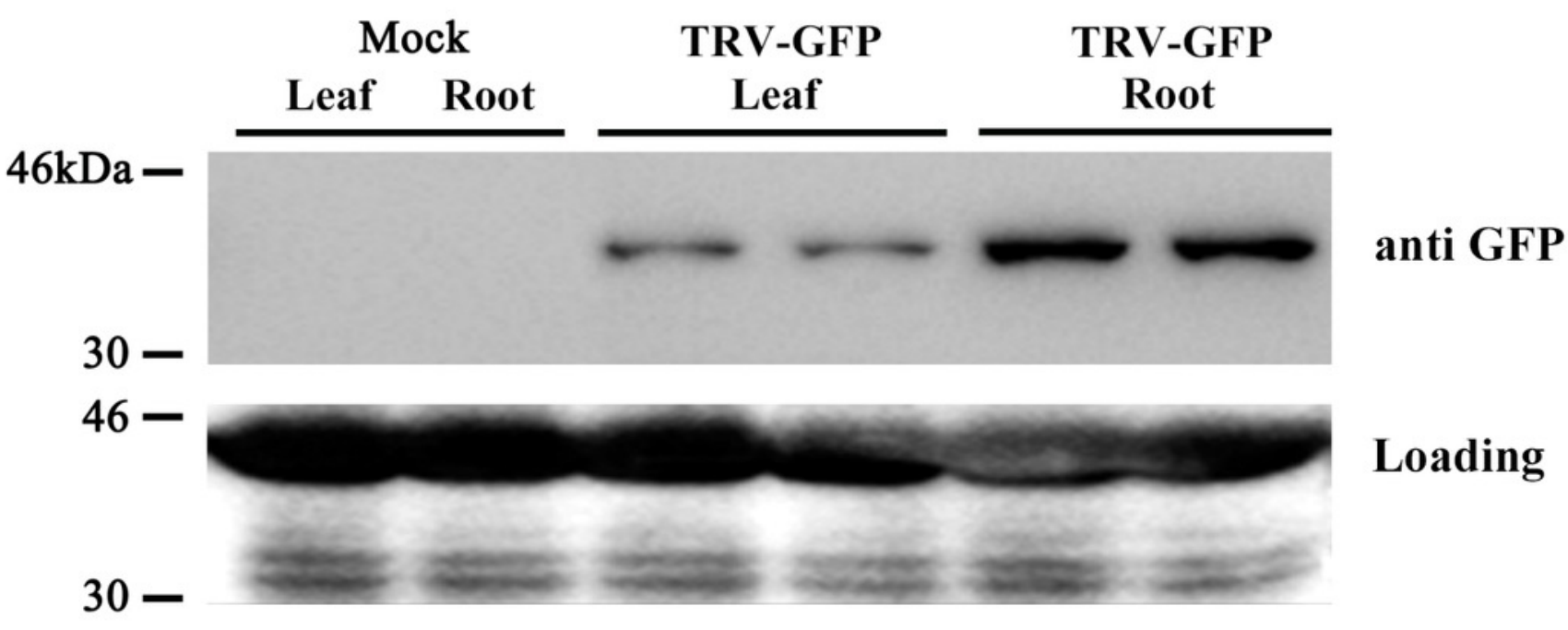

b

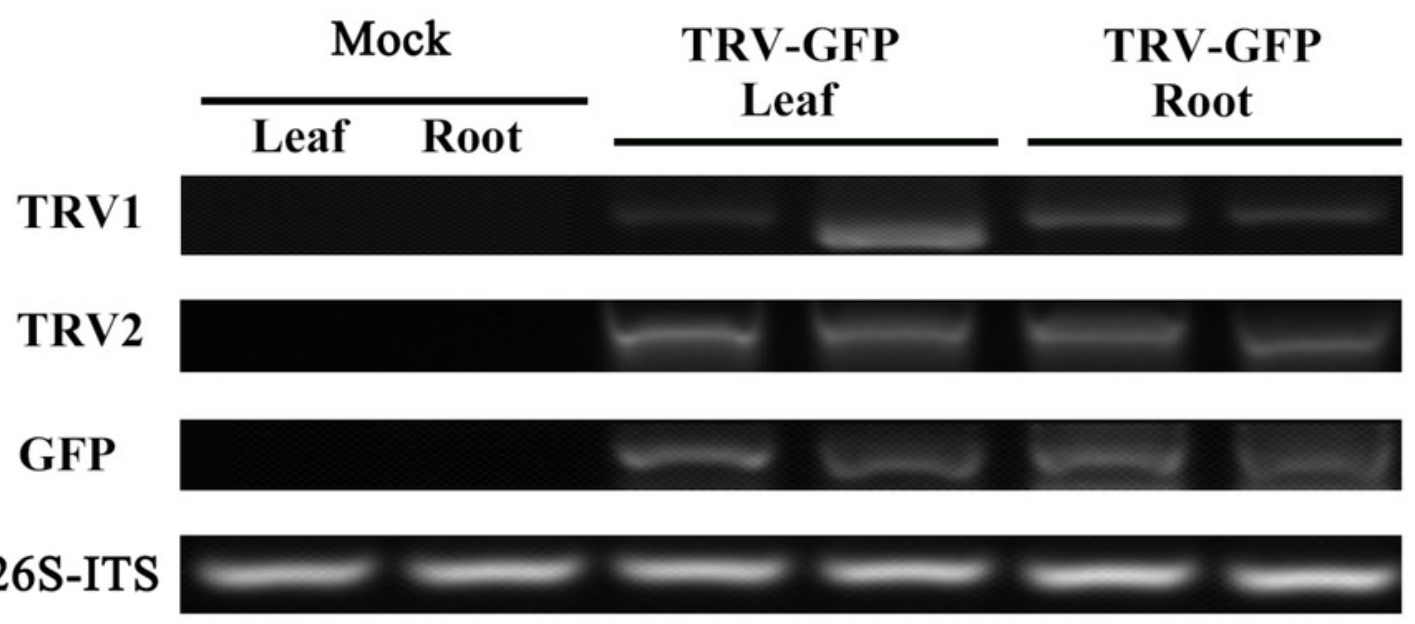

